Eisenstark, A., Goldberg, S. S. \& Bernstein, L. B. (1955). J. gen. Microbiol. $12,402-405$

\title{
Lysogenicity in Xanthomonas pruni
}

\author{
By A. EISENSTARK, S. S. GOLDBERG AND L. B. BERNSTEIN \\ Department of Bacteriology, Kansas State College, Manhattan, Kansas, U.S.A.
}

SUMMARY: An apparently lysogenic strain of Xanthomonas pruni was isolated. A series of experiments was performed to determine whether the isolate was lysogenic or whether it was a case of pseudolysogenesis. The results indicated that it was a true case of lysogenesis. The phage released by the lysogenic organism differed from the one used in its selection. The possible origin of this new phage and its lysogenic host is discussed.

Several types of Xanthomonas pruni phage have been characterized by their differences in plaque morphology, host range, serological properties, in burst times, and in burst sizes (Mandell \& Eisenstark, 1952, 1953; Kirchner, 1954). In the process of isolating variant hosts which might be resistant to one or more of the phage types, a culture was obtained and designated as $\mathrm{H} 15 \mathrm{~L}$, which exhibited the possibility of its harbouring of phage. H1 $5 \mathrm{~L}$ was resistant to all of the $X$. pruni phage types. When dilutions of $\mathrm{H} 15 \mathrm{~L}$ were plated out on a susceptible host, $\mathrm{H}_{1}$, plaques appeared, even though no phage had been added. Such action might have resulted from lysogenesis of $\mathrm{H1} 5 \mathrm{~L}$, or as the result of phage mechanically carried by resistant $\mathrm{H} 1$ cells (pseudolysogenesis). These situations have been discussed in detail by Lwoff (1953). A series of tests was performed to determine whether the phenomenon was due to actual lysogenesis existing in $\boldsymbol{X}$. pruni or to carried phage.

\section{METHODS}

The procedures for plating, titrating phage and preparing media were essentially the same as those described by Adams (1950) for Escherichia coli strain B except that plates were incubated at $24^{\circ}$ rather than $37^{\circ}$.

The two Xanthomonas pruni hosts described in this report are $\mathrm{H} 1 \mathrm{5L}$, the suspected lysogenic culture, and $\mathrm{H1}$, obtained originally from the American Type Culture Collection, no. 10,016, which is susceptible to all of the phage types that have been isolated. The types of phage used in the $X$. pruni phage work have been designated as $\mathrm{Xp1}, \mathrm{Xp2}, \mathrm{Xp3}, \mathrm{Xp4}, \mathrm{Xp5}$ and $\mathrm{Xp} 8$. All of these behave as virulent phages. $\mathrm{Xp} 8$ is the phage that is released by host H1 5L. The suspected lysogenic culture, H1 $5 \mathrm{~L}$, was obtained by plating $\mathrm{H}_{1}$ and an excess of Xp4 phage. After incubation, resistant colonies were selected.

The $\mathrm{Xp} 4$ stock used in these experiments was obtained originally by selection of a particular plaque type from a mixed population. In order to minimize the presence of mutant forms, $\mathrm{Xp}_{\mathrm{p}}$ stocks for each experiment were prepared by selection of isolated typical Xp 4 plaques.

The method described by Adams (1950) was followed in the determination of antiserum inactivation constants. 


\section{RESULTS}

Isolates of suspected lysogenic organisms were restreaked, incubated, harvested and washed in an attempt to rid them of externally carried phage. This procedure was followed through twenty restreakings. Lysis still occurred when re-isolates were plated back on the susceptible host. When susceptible host $\mathbf{H} 1$ was exposed to phage $\mathrm{Xp} 1$ and similarly treated, it lost phage $\mathrm{Xp} 1$ after the second or third restreaking. This is interpreted as evidence that the phage in the suspected lysogenic culture was not carried mechanically; otherwise it would have been lost upon restreaking as was $\mathrm{Xp} 1$.

Antiserum will inactivate extracellular phage but not intracellular. Hence, if culture $\mathrm{H1} \mathrm{5L}$ is truly lysogenic, it should be possible to recover phage even though the culture has been transferred through an antiserum medium. It was found that after seven transfers through antiserum medium, culture H $15 \mathrm{~L}$ continued to release phage.

Cells of $\mathrm{H} 15 \mathrm{~L}$ were plated on plain nutrient agar to get a regular colony count and on nutrient agar seeded with susceptible host to get a plaque count. In the presence of lysogenesis, there would be an approximate 1:1 ratio of colonies to plaques. If the phage were being carried mechanically, the plaque count should exhibit no relationship to the colony count. The data in Table 1 indicate that the plaques probably originated from single lysogenic organisms, rather than from free phage. Although the ratios are not 1:1, there does appear to be a significant relationship between the number of colonies and the number of plaques.

Table 1. Ratio of colonies to plaques when aliquots of $H 15 L$ were plated out on (1) nutrient agar and (2) nutrient agar seeded with host $\mathrm{H} 1$

$\begin{array}{cccc}\text { Sample no. } & \text { Colonies } & \text { Plaques } & \text { Ratio } \\ 1 & 302 & 908 & 1: 3 \cdot 0 \\ 2 & 75 & 290 & 1: 3 \cdot 9 \\ 3 & 37 & 121 & 1: 3 \cdot 3\end{array}$

An additional step was to make replica plates (Lederberg \& Lederberg, 1952) from colonies of $\mathrm{H} 15 \mathrm{~L}$ on to agar plates which had been seeded with susceptible host. A halo developed around each colony that arose on the new plates, indicating lysis. If this were a consequence of carried phage, the results of this replica plating technique would indicate that each bacterial colony had carried phage, an unlikely situation. A more conceivable explanation is that each colony consisted of lysogenic cells.

A comparison of the phage $(\mathrm{Xp} 8)$ arising from the lysogenic host with the phage (Xp4) which had been added to the host in the original isolation process revealed distinct differences. If $\mathrm{Xp} 8$ had been a carried phage, it should have been identical with $\mathrm{Xp} 4$, since $\mathrm{Xp} 4$, was the only phage known to have been in contact with this host. $\mathrm{Xp} 8$ was found to differ from $\mathrm{Xp} 4$ in plaque morphology, as may be seen in Pl. 1 A, B. Almost all of the plaques produced by $\mathrm{Xp} 8$ were cloudy, small, and irregular, while $\mathrm{Xp} 4$ plaques were much larger, 


\section{$404 \quad$ A. Eisenstark, S. S. Goldberg and L. B. Bernstein}

clear, with a distinct halo. In addition to the cloudy plaques, a small number of larger and clear plaques were produced by Xp8. A few may be seen in Pl. $1 B$. Studies are in progress to determine the origin and significance of this second plaque type.

$\mathrm{Xp} 8$ differs from $\mathrm{Xp} 4$ in its host range, as may be seen in Table 2. A series of isolates has been collected which are resistant to one or more of the $\mathbf{X p}$ series of phages. These were used to show that the host range of $\mathbf{X p 4}$ and Xp 8 differed.

Table 2. Host range differences

$\begin{array}{lll}\text { Host } & \text { Xp4 phage } & \text { Xp8 phage } \\ \text { H1 } & \text { Lysed } & \text { Lysed } \\ \text { H1 5L } & \text { Not lysed } & \text { Not lysed } \\ \text { H80 } & \text { Lysed } & \text { Not lysed } \\ \text { H23 } & \text { Not lysed } & \text { Lysed } \\ \text { H8 } & \text { Not lysed } & \text { Lysed } \\ \text { H204 } & \text { Not lysed } & \text { Lysed }\end{array}$

In order to test the possibility that $\mathrm{Xp} 4$ stock might contain a small number of contaminant $\mathrm{Xp} 8$ phage, $\mathrm{Xp} 4$ was plated on host $\mathrm{H23}$, which is resistant to $\mathrm{Xp} 4$ but susceptible to $\mathrm{Xp} 8$. In the plating of $10^{10} \mathrm{Xp} 4$ phage not a single plaque arose, indicating that if the $\mathrm{Xp} 4$ stock was contaminated with $\mathrm{Xp} 8$, the contaminant must be present in a frequency less than one out of $10^{10}$.

In addition to the above comparisons of $\mathrm{Xp} 4$ and $\mathrm{Xp} 8$, immunological data were also obtained. Immunologically it was found that $\mathrm{Xp} 4$ and $\mathrm{Xp} 8 \mathrm{do}$ not fall into distinct groups, although they are not inactivated at equal rates by $\mathrm{Xp} 4$ antiserum. The inactivation constant for the homologous system, Xp4 phage and $\mathrm{Xp} 4$ antiserum, was found to be $K=95 \cdot 6$, whereas the constant for $\mathrm{Xp} 8$ phage and $\mathrm{Xp} 4$ antiserum was $K=61 \cdot 5$.

If the phenomenon in question resulted from a carried phage, it should be possible to demonstrate it with any of the $\mathrm{Xp}$ phages. $\mathrm{H} 1 \mathrm{LL}$ is resistant to all of the Xp series and if it were merely a selected culture that was carrying phage, it should not make any difference which $X p$ phage was used in the selection process. However, it was found that $\mathrm{Xp} 4$ was the only one of the series that enabled the isolation of $\mathrm{H} 15 \mathrm{~L}$. In the process of selecting hundreds of resistant organisms, about $25 \%$ of the isolates from Xp4 plates possessed the properties of $\mathrm{H} 1 \mathrm{5L}$, whereas no isolate from plates seeded with $\mathrm{Xp1}$, $\mathrm{Xp2}, \mathrm{Xp3}$ and Xp5 exhibited similar properties.

\section{DISCUSSION}

Ordinarily, when dilutions of lysogenic bacteria are plated on a susceptible host, the plaques that arise exhibit a central colony inasmuch as the lysogenic bacterium itself grows into a distinctly visible colony. An occasional organism within a colony bursts and releases phage, hence the plaque around the colony. When H1 5L was plated on $H_{1}$, central colonies could be seen in only a small percentage of plaques, and then only indistinctly. Possibly the plaque 

Journal of General Microbiology, Vol. 12, No. 3

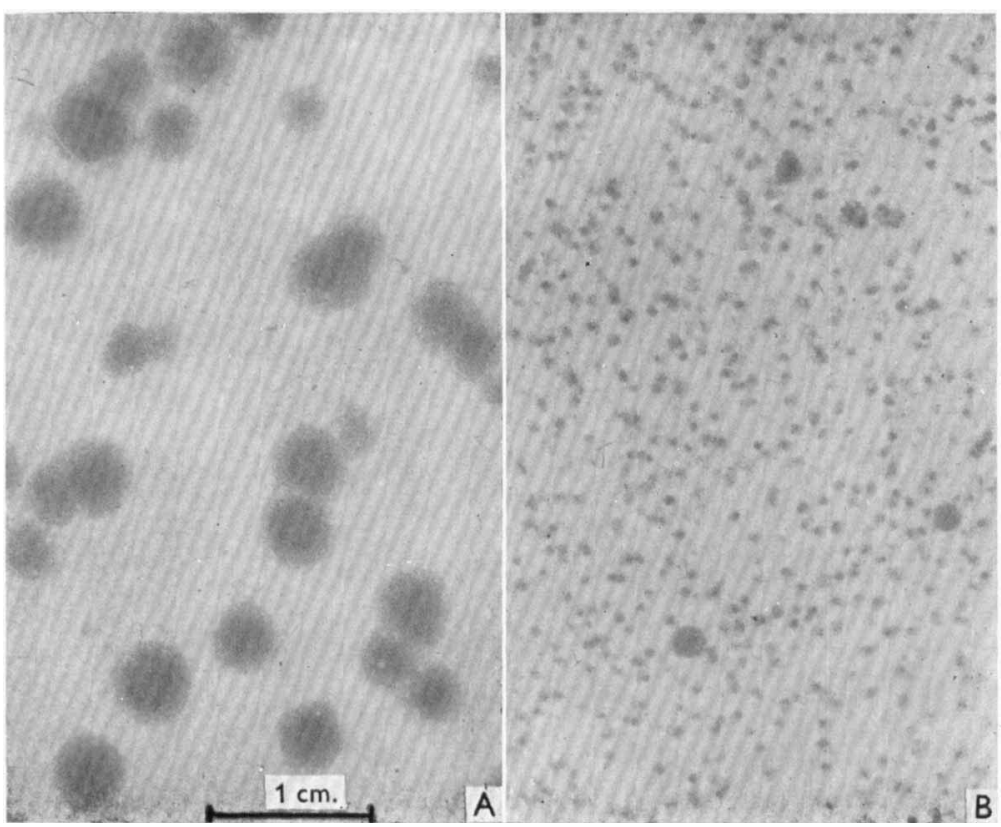

A. Fisenstark, S. S. Goldberg \& L. B. Bernstein-I.ysogenicity in Xanthomonas prunt. PIate 1

(Facing $p .405)$ 
itself was so turbid and small that the central colony was obscured by this growth throughout the plaque.

The results of this investigation pose several questions relative to the origin of $\mathrm{H} 1 \mathrm{LL}(\mathrm{Xp} 8)$. Does $\mathrm{H} 1$ actually contain a small number of $\mathrm{H} 15 \mathrm{~L}(\mathrm{Xp} 8)$ cells, and $\mathrm{Xp} 4$ merely select these by lysing the remainder of the population? This seems unlikely in the light of the above experiments where $\mathrm{Xp1}, \mathrm{Xp2}$, $\mathrm{Xp3}$ and Xp5 all failed to act as selecting agents.

Are some of the $\mathrm{Xp} 4$ phage able to behave as temperate phage that invade $\mathrm{H} 1$ cells and cause them to be lysogenized? This would seem to be a possible explanation since lysogenization is a common occurrence among other phagehost systems (Lwoff, 1953). However, if this is the case, why is the phage that is released by $\mathrm{H} 1 \mathrm{5L}(\mathrm{Xp8})$ apparently different from $\mathrm{Xp4}$ ? A possible explanation, that $\mathrm{Xp} 4$ is not pure and contains a small proportion of temperate $\mathrm{Xp} 8$ phage capable of lysogenizing host cells, seems unlikely since no plaques arose when $10^{10} \mathrm{Xp4}$ phage were plated out on $\mathrm{H} 23$ which is resistant to $\mathrm{Xp} 4$ but susceptible to $\mathrm{Xp8}$. It is also possible that $\mathrm{Xp} 8$ represents a hostinduced modification (Luria; 1953) of $\mathrm{Xp} 4$ which actually has changed its characteristics after a multiplication cycle within its host. $\mathbf{A}$ third possible explanation is that the Xanthomonas pruni cells harbour Xp8 prophage, but that the mature phage are not developed until the bacterial cells are stimulated to do so by $\mathrm{Xp} 4$ nucleic acid. These explanations are in need of careful experimental examination.

Contribution no. 301, Department of Bacteriology, Kansas Agricultural Experiment Station, Manhattan. These studies were aided by a contract between the Office of Naval Research, Department of the Navy, and Kansas State College, NR 135-172.

\section{REFERENCES}

Adams, M. H. (1950). Methods of study of bacterial viruses. Meth. med. Res. pp. 1-73.

KIRChNER, C. E. J. (1954). The antigenic relationships of bacteriophages active against Xanthomonas pruni. Thesis. Kansas State College.

Lederberg, J. \& LeDerberg, E. M. (1952). Replica plating and indirect selection of bacterial mutants. J. Bact. 63, 399.

Luria, S. (1953). Host-induced modifications of viruses. Cold Spr. Harb. Symp. quant. Biol. 18, 237.

LwoFf, A. (1953). Lysogeny. Bact. Rev. 17, 269.

MANDELl, J. D. \& Eisenstark, A. (1952). Bacterial viruses active against Xanthomonas pruni. Monthly Research Report, Office of Naval Research, April 1952.

Mandell, J. D. \& Eisenstark, A. (1953). Factors affecting plaque formation by Xanthomonas pruni bacteriophage. Phytopathology, 43, 27.

\section{EXPLANATION OF PLATE}

A. Plaques produced by Xp4. These are large and clear with a distinct halo.

B. Plaques produced by $\mathrm{Xp8}$, the bacteriophage released by lysogenic cells of $X$ anthomonas pruni. These are minute and cloudy. There is always a small percentage of larger plaques. A few of these plaques may be seen in the figure.

(Received 24 September 1954) 